\title{
SEVERIDAD DE CURVULARIA EN 67 LÍNEAS AUTOFECUNDADAS S4 DE MAÍZ AMARRILLO
}

\author{
${ }^{\bullet}$ Felipe Rafael Garcés Fiallos ${ }^{1,2}$, Ángel Jefferson Aguirre Calderón ${ }^{1}$, Jaime Javier Carbo Morán ${ }^{1}$, \\ Gabriel Antonio Liubá Delfini ${ }^{1}$ \\ ${ }^{1}$ Unidad de Investigación Científica y Tecnológica, Universidad Técnica Estatal de Quevedo, km 7 1/2 vía Quevedo-El Empalme, \\ C. P. 73. Mocache,Los Ríos, Ecuador. ${ }^{\circ}$ felipegarces23@yahoo.com \\ ${ }^{2}$ Facultad de Ciencias Agrarias, Escuela de Ingeniería Agronómica, Universidad Técnica Estatal de Quevedo, Campus Ing. \\ Manuel Haz Álvarez, km 1 11/2 vía Quevedo-Santo Domingo de los Tsáchilas, C. P. 73. Quevedo, Los Ríos, Ecuador
}

RESUMEN

口 ntre los principales problemas que afectan la Eproducción del cultivo de maíz (Zea mays L.), podemos citar a las enfermedades, causadas por diferentes patógenos, entre ellos la mancha foliar de curvularia causada por Curvularia spp. El control más barato, viable y que no causa daños al medio ambiente contra casi cualquier patógeno, es a través de la resistencia genética de la planta hospedante es decir, mediante el mejoramiento genético y utilización de variedades resistentes. Por lo tanto, el objetivo de esta investigación fue evaluar la severidad de curvularia en líneas promisorias autofecundadas S4 de maíz amarillo, obtenidos a partir de materiales comerciales. En la primera población (LM1) se encontraron valores máximos de 5.6 y menores de 1.4 lesiones $\mathrm{cm}^{-2}$, mientras que en la segunda población (LM2) los promedios máximos y mínimos fueron de 8.0 y 1.5 lesiones $\mathrm{cm}^{-2}$, respectivamente. Se constató diferencia entre las líneas evaluadas en función de la severidad de mancha de curvularia, en los dos experimentos. Con la información obtenida se torna importante considerar los valores de severidad obtenidos en estos experimentos, para el futuro desarrollo de nuevos híbridos de maíz con caracteres contrastantes en el comportamiento de la planta a determinadas enfermedades, asegurando el éxito de un programa de mejoramiento genético. Por otro lado, para futuros trabajos similares se recomienda realizar más de una evaluación y en diferentes estadios fenológicos del cultivo.

Palabras claves: Zea mays, evaluación sanitaria, Curvularia spp.
Abstract

mong the main problems affecting the production of corn(Zeamays L.), it could bementioned the diseases caused by different pathogens, including Curvularia leaf spot caused by Curvularia spp. The cheaper control, viable and that does not cause any environmental damage against almost any pathogen is through genetic host resistance, i.e. by breeding and use of resistant varieties. Therefore the aim of this study was to evaluate the severity of curvularia in promissory inbred lines S4 of yellow maize derived from commercial materials. In the first population (LM1) found maximum values of 5.6 and of minimum 1.4 under injuries by $\mathrm{cm}^{-2}$, while in the second experiment (LM2) maximum and minimum averages were 8.0 and 1.5 lesions by $\mathrm{cm}^{-2}$, respectively. Difference was found among the valuated line depending on the severity of Curvularia leaf spot, in both experiments. With the information obtained, it becomes important to consider the severity values obtained in these experiments, for the future development of new maize hybrids with contrasting characters in the behavior of the plant to certain diseases, ensuring the success of a breeding program. On the other hand, for future similar work it is recommended to do more than one evaluation during different phenological stages of the crop.

Key words: Zea mays, sanitary assessment, Curvularia spp.

\section{INTRODUCCIÓN}

$\mathrm{L}_{\mathrm{a}}^{\mathrm{a}}$ a superficie mundial de maíz (Zea mays L.) en el período 2007-2008 llegó a 159.9 millones de hectáreas, mientras que entre el 2008-2009 hubo

Recibido: 4-Julio-2011. Recibido en forma corregida: 31-Octubre-2011. Aceptado: 24-Noviembre-2011.

Publicado como NOTA TÉCNICA en Ciencia y Tecnología 4(2):39-44. 2011 una ligera disminución de 2.4 millones de hectáreas sembradas, por lo que la superficie total llegó a las 157.5 millones de hectáreas. De este total Estados 
Unidos engloba la mayor parte con 31.83 millones de hectáreas que representa el 19\% y le sigue China con 29.8 millones de hectáreas (18\% del total) (MAGAP, 2007).

El maíz amarillo duro en el Ecuador es uno de los productos agrícolas más importantes de la economía nacional. Constituye la principal materia prima para la elaboración de alimentos concentrados (balanceados) destinados a la industria animal, especialmente a la avicultura comercial, que es una de las actividades más dinámicas del sector agropecuario del país (Villavicencio y Zambrano, 2009).

Según el MAGAP (2009) entre las provincias de Guayas, Los Ríos, Manabí y Loja, suman una superficie de maíz de 228,761 ha. En la provincia de Los Ríos se encuentra concentrada el 56\% del total de la producción nacional estimada para el 2009.

Entre los principales problemas que afectan la producción podemos citar a las enfermedades, causadas por diferentes patógenos (bacterias, hongos, nemátodos, virus y fitoplasmas). Reis et al. (2004) relata como agentes causantes de enfermedades en el cultivo del maíz, a organismos biotróficos y necrotróficos. Entre estos últimos se encuentra la mancha de curvularia causada por el hongo Curvularia spp. Este fitopatógeno pertenece a la Clase de los Deuteromicetes, Orden Moniliales y Familia Dematiacea (Alexopolus et al., 1996). Este género actualmente está compuesto por más de 40 taxones (Sivanesan et al., 2003), pudiendo infectar a las gramíneas Eleusine indica (L.) Gaertn. subsp. indica, Panicum maximum Jacq., Saccharum officinarum L., Setaria verticillata (L.) P. Beauv., Sorghum halepense (L.) Pers. y Zea mays (Lima y Furtado, 2007). Según Shurtleff (1992) existen varias especies, entre las cuales están C. lunata (Wakker) Boed var. aeria, C. pallescens Boed, C. inaequalis (Shear) Boed, C. maculans (Bancroft) Boed, $C$. intermedia Boed, C. clavata Jain, C. tuberculata Jain, C. eragrostidis (P. Henn.) J. A. Meyer y C. senegalensis (Speg.) Subram. La especie que prevalece en este cultivo es C. lunata (Akinbode, 2010). Reis et al. 2004, menciona que esta enfermedad puede causar daños significativos en el cultivo de maíz.

Las enfermedades pueden causar daños y pérdidas en cualquier cultivo. Según Casa et al. (2004), los daños asociados a las enfermedades foliares son consecuencia del mal funcionamiento y destrucción de los tejidos fotosintéticos debido al aumento del número de lesiones, que pueden determinar la necrosis de toda la hoja. La necrosis y muerte prematura de las hojas limita la intersección de la radiación solar y la translocación de fotoasimilados al desarrollo del grano.

Para contrarrestar las enfermedades, una de las medidas de control es el genético. Menciona
Vallejo y Estrada (2002) que este tipo de control de las enfermedades, basado en la utilización de cultivares resistentes, es el método más eficaz y económico en la medida que suprime o disminuye la aplicación de pesticidas durante el cultivo, aminora la contaminación de las cosechas, del medio ambiente y los riesgos para la salud humana; además de reducir los costos de producción de los cultivos tornándolos competitivos y sostenibles. Por otro lado, Van Der Plank (1968) relata que las enfermedades reducen el rendimiento y la calidad, por lo que la resistencia a enfermedades es necesaria e importante. También Agrios (2004) describe que el método de mejoramiento más común de la resistencia del hospedante contra casi cualquier patógeno, es a través de la resistencia genética de la planta hospedante es decir, mediante el mejoramiento genético y utilización de variedades resistentes.

Por lo tanto, el objetivo de esta investigación fue evaluar la severidad de curvularia en 67 líneas promisorias de maíz amarillo autofecundadas S4, obtenidos por aptitud combinatoria general.

\section{Materiales y MéTOdos}

E ste trabajo se desarrolló durante la época seca del año 2010, en la Finca Experimental La María de la UTEQ, ubicada en el km 7 1/2 vía Quevedo-El Empalme, cuyas coordenadas geográficas son las siguientes: $79^{\circ}$ $27^{\prime}$ de longitud oeste y $01^{\circ} 06^{\prime}$ de latitud sur, a una altura de $73 \mathrm{msnm}$. Las condiciones agro-meteorológicas del sitio experimental se detallan a continuación: temperatura media anual de $24.5^{\circ} \mathrm{C}$, humedad relativa de $84.0 \%$, heliofanía de 75.2 horas luz mes $^{-1}$ y una precipitación media anual de 2,178 $\mathrm{mm}$.

Fueron realizados dos experimentos, utilizándose 42 líneas de la población uno (LM1) (primer experimento) y 25 líneas de la población dos (segundo experimento), ambas procedentes de híbridos comerciales, sumando en total 67 materiales. Estos materiales son líneas S4 (obtenidas por autofecundación a través del método de selección masal). Se sembraron ambos experimentos el 04-06-2010 con las siguientes especificaciones de siembra: tamaño de la parcela: $5 \mathrm{~m}$ de largo x $3.60 \mathrm{~m}$ de ancho, sumando una superficie de $18.0 \mathrm{~m}^{2}$; tamaño de la parcela útil: $5 \mathrm{~m}$ de largo x 1.8 $\mathrm{m}$ de ancho lo que da una superficie de $9 \mathrm{~m}^{2}$; número de hileras: 4; distancia entre hileras: $0.90 \mathrm{~m}$; distancia entre plantas: $0.20 \mathrm{~m}$; plantas por hilera: 25 ; plantas por parcela: 100 y una población por hectárea de 55,555 plantas. Las semillas fueron tratadas previamente con carboxin + captan en una dosis de 100 g por cada 100 $\mathrm{kg}$ de semilla. Para el control de plagas fue utilizado clorpirifos, cipermetrin y carbofuran con dosis de 200 $\mathrm{cm}^{3} \mathrm{ha}^{-1}, 30 \mathrm{~kg}$ y $250 \mathrm{~g}$ en $50 \mathrm{~kg}$ de arena por hectárea 
respectivamente. Para el control de enfermedades no fue aplicado ningún fungicida, con la finalidad de observar la respuesta fitosanitaria de las plantas sin injerencia de este factor (fungicida).

La evaluación de la severidad de mancha de curvularia (Curvularia spp.), fue realizada el 0508-2010 a los 93 días después de la siembra, siendo contemplada solamente una evaluación fitosanitaria en el estadío fenológico R8 conforme la escala de Ritchie y Hanway (1982). Fueron extraídas tres hojas por cada planta (en dos plantas por cada tratamiento) correspondientes a los estratos inferior, medio y superior para el respectivo análisis de las mismas. La fitopatometría utilizada fue objetiva, cuantificando el número de lesiones $\mathrm{cm}^{-2}$, utilizando para ello un estéreo microscopio marca SWIFT OPTICAL INTRUMENTS. INC. modelo SM90. Cabe mencionar que se realizó la evaluación de severidad porque según Bergamin Filho y Amorim (1996) relatan que para medir enfermedades foliares como royas, oídios, míldiús y manchas, el porcentaje de tejido cubierto por síntomas retrata mejor la cantidad de enfermedad que la incidencia.

El delineamiento experimental utilizado en estos experimentos, fue un diseño de bloques completos al azar con tres repeticiones. Los valores de la severidad fueron sometidos a un análisis de variancia y sus promedios comparados a través de la prueba de Tukey al $5 \%$ de probabilidad del error. Para el análisis estadístico fue utilizado el software SAS 9.0 (SAS, 2002).

\section{RESUltados Y DISCUSión}

T os síntomas de la mancha por curvularia (Figura 1) se manifestaron como manchas circulares con halo externo amarillento característico de mancha de curvularia, iniciando con pequeñas manchas hasta llegar a cubrir toda la superficie. La descripción de los síntomas antes mencionados coinciden con las

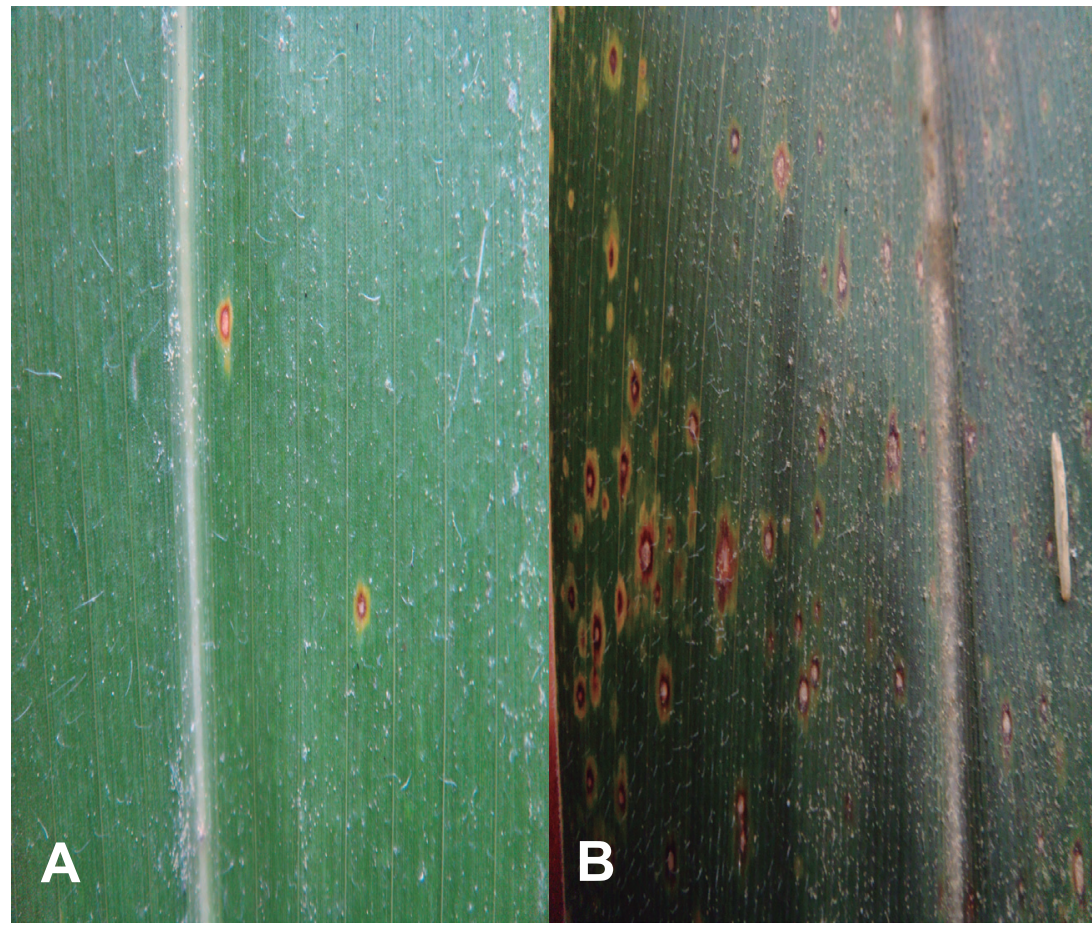

Figura 1. Manchas circulares con halo externo amarillento característico de curvularia, iniciando con pequeñas manchas (A), hasta llegar a cubrir toda la superficie foliar (B).

realizadas por Malaguti y Subero (1971), Shurtleff (1992), Reis et al. (2004) y Varón y Sarria (2007).

En el primer experimento (LM1) se encontraron valores máximos de 5.6 y menores de 1.4 lesiones $\mathrm{cm}^{-2}$ (Figura 2) y no se encontraron diferencia estadística significativa (0.005), mientras que en el segundo experimento (LM2) los promedios máximos y mínimos fueron de 8.0 y 1.5 lesiones $\mathrm{cm}^{-2}$, respectivamente, (Figura 3) encontrándose diferencia estadística significativa (0.005). Estas diferencias entre cultivares son relatadas en Córdoba, Argentina por Arrieta et al. (2007) donde evaluando la incidencia, distribución espacial y severidad de enfermedades foliares entre ellas la de curvularia, encontraron algunos genotipos entre 11 evaluados, con valores de severidad (\%) de 1 (la enfermedad se presenta en las plantas sin 


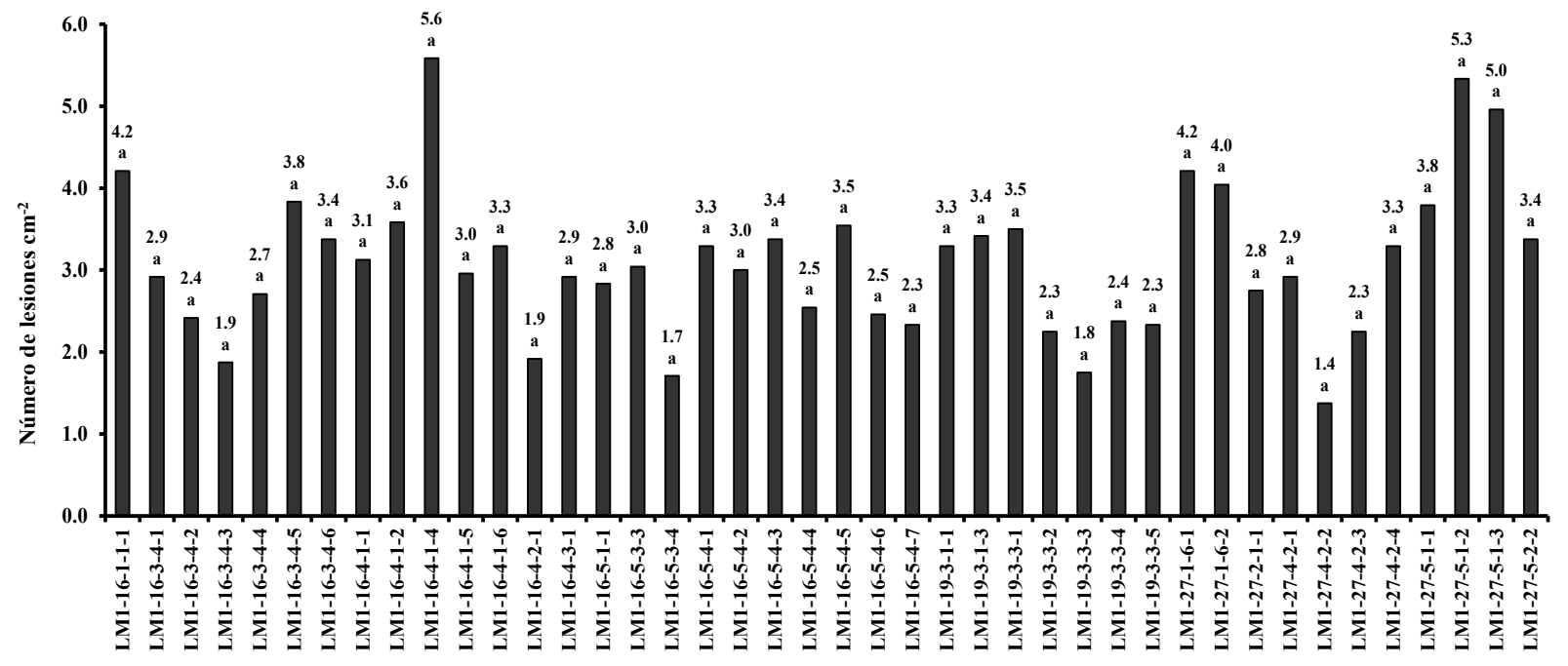

Híbridos promisorios de maíz amarrillo LM1

Figura 2. Número de lesiones $\mathrm{cm}^{-2}$ de mancha de curvularia en hojas de 42 líneas promisorias de maíz LM1. Finca Experimental La María. Quevedo, Ecuador. CV: $\mathbf{3 4 . 4 5 \% . ~}$

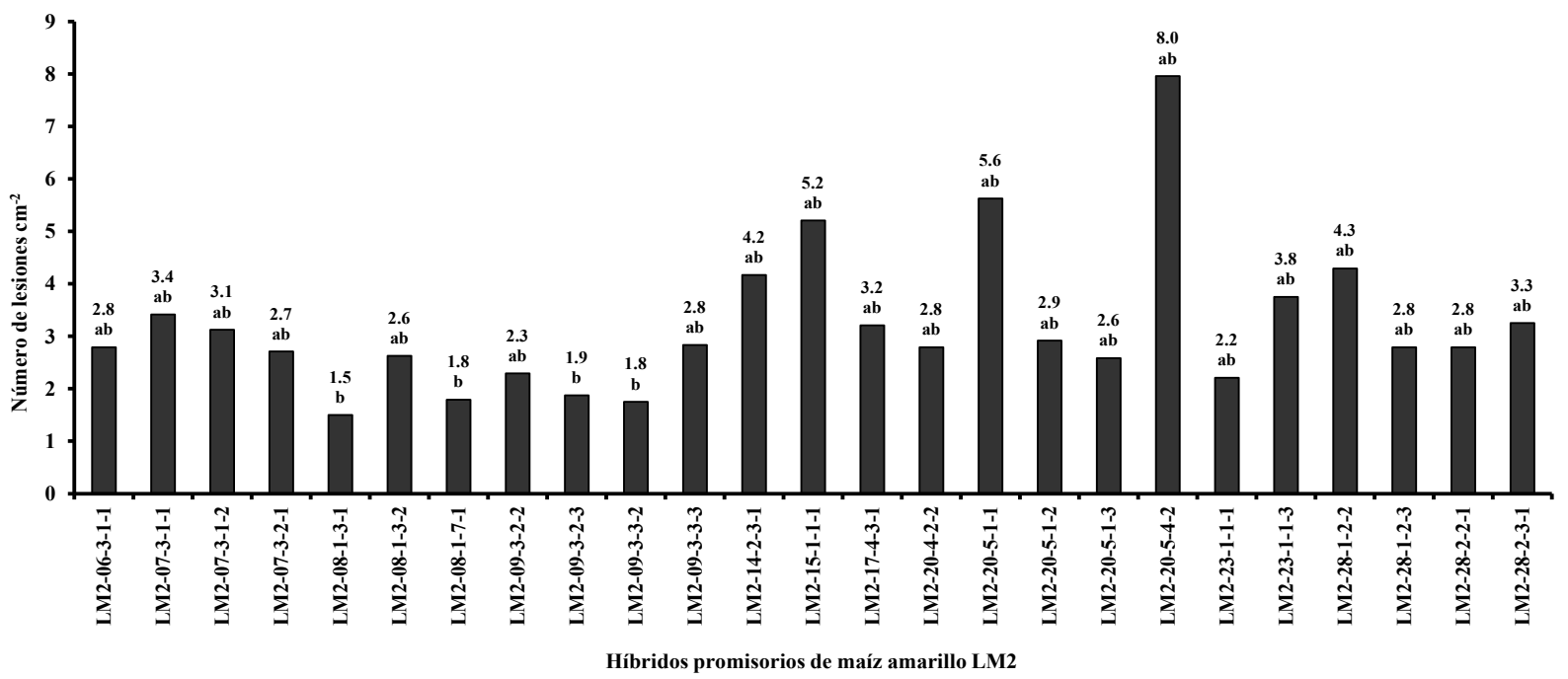

Figura 3. Número de lesiones $\mathrm{cm}^{-2}$ de mancha de curvularia en hojas de 25 líneas promisorias de maíz LM2. Finca Experimental La María. Quevedo, Ecuador. CV: 34.92\%.

causar daño económico), según esos autores. Caso similar fue encontrado en Centro América en genotipos de maíz, encontrándose bajos porcentajes de pudrición de mazorca (4-10\%), lo cual indica que existen genotipos tolerantes a este factor adverso de origen biótico, si se considera que la pudrición de mazorca como la enfermedad de mayor importancia económica en Honduras, Costa Rica y Guatemala (Fuentes et al., 1993).

La mancha foliar de curvularia en estos experimentos fue evaluada a los 93 días después de la siembra, en el estadio R8, diferentemente de lo realizado por Arrieta et al. (2007), quien evaluó entre los 80 a 85 días encontrando 10 a $30 \%$ de área foliar afectada.
Sin embargo, el mismo autor menciona que esto podría repercutir sustancialmente en los rendimientos debido a que la planta está llegando a su madurez fisiológica y las hojas bajeras son las más afectadas.

Cabe mencionar que no existen trabajos sobre la mancha de curvularia, donde hayan utilizado esta metodología de cuantificación (número de lesiones $\mathrm{cm}^{-2}$ ) de la enfermedad. No obstante, trabajos como los de Carvalho et al. (2010), Lopes-Cancellier et al. (2010) y Vaz-de-Melo et al. (2010), usaron escalas arbitrarias para la cuantificación de la enfermedad, poniendo de manifiesto la importancia del presente trabajo como pionero en este tipo de investigaciones.

Aunque fue objeto de estudio la mancha foliar 
de curvularia, se constató también otras enfermedades foliares como helmintosporiosis (Bipolaris spp.), mancha de diplodia (Stenocarpella macrospora (Earle) Sutton) y cinta roja (Spiroplasma). Malaguti y Subero (1971) relatan que muchas veces se encuentran asociadas las manchas de curvularia y helmintosporiosis. Esto hecho se constató en este experimento.

\section{Conclusiones}

$\mathrm{E}^{\mathrm{n}}$ el primer experimento (LM1) se encontraron valores entre 1.4 y 5.6 lesiones $\mathrm{cm}^{-2}$, mientras que en el segundo experimento (LM2) promedios de $1.5 \mathrm{a}$ 8.0 lesiones $\mathrm{cm}^{-2}$.

Se constató diferencia entre las líneas evaluadas en función de la severidad de mancha de curvularia en los dos experimentos, y diferencia estadística en el segundo.

\section{RECOMENDACIONES}

$\mathrm{C}$ on la información obtenida se torna importante considerar los valores de severidad obtenidos en estos experimentos, para el futuro desarrollo de nuevos híbridos de maíz con caracteres contrastantes en el comportamiento de la planta a determinadas enfermedades, asegurando el éxito de un programa de mejoramiento genético. Por otro lado, para futuros trabajos similares se recomienda realizar más de una evaluación y en diferentes estadios fenológicos del cultivo.

\section{LiteratURA CITADA}

Agrios, G. N. 2004. Fitopatología. Segunda edición. Limusa. México DF.

Akinbode, O. A. 2010. Evaluation of antifungal efficacy of some plant extracts on Curvularia lunata, the causal organism of maize leaf spot. African Journal of Environmental Science and Technology 4(11):797-800.

Alexopoulos, G. J., C. W. Mims and M. Blackwell. 1996. Introductory Mycology (4th Ed.). Jhon Wiley e Sons, New York, USA.

Arrieta, K., C. Salazar, R. Ocampo, y N. Villarreal. 2007. Enfermedades patogénicas en los híbridos de maíz (Zea mays) en el medio y bajo sinú del Departamento de Córdoba. Temas Agrários.12(1):58-69.

Bergamin Filho, A. e L, Amorim. 1996. Doenças de Plantas Tropicais: epidemiologia e controle econômico. Ceres, São Paulo, SP, Brasil.

Carvalho, V. de. E., F. S. Afférri, M. A. Dotto, A. Reis da Silva, A. Vaz de Melo y R. B. Capellesso. 2010.
Avaliação agronômica e de sanidade em genótipos de milho no sul do Tocantins, em duas épocas de semeadura. Científica 38(1-2):39-49.

Casa, R. T., E. M. Reis, e M. M. C. Blum. 2004. Quantificação de danos causados por doenças de milho. Em: I Workshop de Epidemiologia de Doenças de Plantas. Resumo. Viçosa, MG, Brasil.

Fuentes, M., J. L. Quemé y C. Pérez. 1993. Efectos de aptitud combinatoria general e identificación de híbridos triples de maíz (Zea mays L.) grano blanco. Programa Regional de Maíz (PRM), 1991. Agronomía Mesoamericana 4:71-76.

Lima, A. e M. Furtado. 2007. Espécies do género Curvularia (fungos anamórficos: Hyphomycetes) na ilha de Santiago, Cabo Verde. Portugaliae Acta Biol. 22:145-156.

Lopes-Cancellier, L., V. de. E. Carvalho, F. S. Afférri, R. C. Costa-Bachega e J. M. Peluzio. 2010. Potencial de uso in natura e sanidade de linhagens de milho uft em top cross Revista Verde 5(4):103-112.

Malaguti, G. y L. Subero. 1971. Manchas foliares del maíz causadas por curvularia en venezuela. Centro de Investigaciones Agronómicas. Maracay.

MINISTERIO DE AGRICULTURA, GANADERÍA, ACUACULTURA Y PESCA (MAGAP). 2007. Panorama mundial de maíz amarillo. (en línea). Consultado 17 Sept. 2010. Disponible en http:// sigagro.flunal.com/index.php?option $=$ com_wrap per\&view $=$ wrapper\&Itemid $=94$.

MINISTERIO DE AGRICULTURA, GANADERÍA, ACUACULTURA Y PESCA (MAGAP). 2009. Pronóstico de producción de maíz y arroz: campaña de invierno 2009. Boletín técnico. 6 pp. (en línea). Consultado 17 Sept. 2010. Disponible en http:// sigagro.flunal.com/ index.php?option $=$ com_conte nt\&view=article\&id=79\&Itemid=99.

Reis, E. M., R. T. Casa e A. C. B. Reis. 2004. Manual de Diagnose e Controle de Doenças de Milho. Graphel. Lages, RS., Brasil.

Ritchie, S. W. and J. J. Hanway. 1982. How a corn plant develops. Iowa State University of Science and Technology. Cooperative Extension Services, Ames, Iowa. Special Report 48.

SAS/STAT® Versão 9.0 del sistema SAS para Windows, copyright 2002 SAS Institute Inc., Cary, NC, USA.

Shurtleff, M. C. 1992. Compendium of Corn Diseases. Second edition. APS. University of Illinois. Urbana, USA.

Sivanesan, A., L. John, J. Alcorn and R. Shivas. 2003. Three new graminicolous species of Curvularia (anamorphic fungi) from Queensland, Australia. Aust. Syst. Bot. 16:275-278.

Vallejo, F. A. C. y E. I. S. Estrada. 2002. Resistencia genética de plantas a enfermedades. En:

Ciencia y Tecnología. 2011. 4(2): 39-44 
Garcés et al.

Mejoramiento Genético de Plantas: 323-343. Universidad Nacional de Plantas. Sede Palmira, Colombia.

Van der Plank, J. E. 1968. Disease Resistance in Plants. First edition. Academic Press. New York \& London.

Varón, F. y V. G. A. Sarria. 2007. Enfermedades del Maíz y su Manejo. Instituto Colombiano Agropecuario. Palmira, Colombia.

Vaz-de-Melo, A., F. S. Afférri, M. A. Dotto, J. M. Peluzio, G. Rodrigues dos Santos e V. de. E. Carvalho. 2010. Reação de híbridos de milho à Curvularia ssp, sob dois níveis de adubação com nitrogênio, no Sul do Tocantins. Scientia Agraria 11(2):149-154.

Villavicencio, P. y J. L. Zambrano. 2009. Guía para la producción de maíz amarillo duro, en la zona central del Litoral Ecuatoriano. INIAP, Estación Experimental Tropical de la zona central del Litoral Ecuatoriano. Boletín $\mathrm{N}^{\circ}$ 353. Quevedo, Ecuador. 